\title{
Judgment accuracy in pair-distance estimation and map sketching
}

\author{
STEPHEN M. KERST, JAMES H. HOWARD, JR., and LEO J. GUGERTY \\ The Catholic University of America, Washington, DC
}

\begin{abstract}
Intersite distances were obtained using both subjective pair-distance judgments and map sketching, either perceptually with the map in view or from memory following a study period. The perceptual distance estimates were more accurate than those made from memory. In addition, when responses were made from memory, intersite distances on the sketched map were more accurate than the same distances estimated in the pair judgment task.
\end{abstract}

Distance estimation from maps is fundamental to a wide variety of navigation tasks. The present experiment compares the relative accuracy of distance judgments obtained using two methods. In the pair-distance method, participants estimated the distance between all possible pairs of a number of map sites, whereas in the direct mapping method, participants simply sketched the map.

The pair-distance method has high face validity since, in applied settings, individuals are often required to estimate the distance between two points (e.g., the present location and a projected destination). A number of previous studies have examined the judgments made with this method using either direct psychophysical scaling (Ekman \& Bratfisch, 1965) or multidimensional scaling (Baird, Merrill, Tannenbaum, 1979; Golledge \& Zannaras, 1973; Howard \& Kerst, 1981). In all of these studies, the subjective distance estimates have been shown to be generally accurate.

In one study, Baird (1979) compared maps derived from pair-distance judgments using multidimensional scaling with those produced by direct mapping or sketching. Although both methods revealed maps that were congruent with the original, his participants judged the direct maps to be more accurate than the derived maps. In an earlier study, MacKay (1976) observed that sketched maps were more accurate than pair-distance maps. On the basis of these findings, Baird (1979) has argued that direct mapping may ultimately prove more useful in assessing spatial knowledge.

The present experiment compares the accuracy of distance judgments using the pair-distance and direct mapping methods when spatial knowledge is based on inspection of a particular map, rather than on general geographic

\footnotetext{
This research was supported by grants from the U.S. Army Research Institute and the National Institute of Mental Health to The Catholic University of America. The authors thank James A. Ballas and Darlene V. Howard for their comments on an earlier version of the manuscript and David Russo for his contribution to the research. Requests for reprints should be addressed to Stephen M. Kerst, Department of Education, The Catholic University of America, Washington, DC 20064.
}

knowledge (MacKay, 1976) or on firsthand familiarity with an area (Baird, 1979).

In the pair-distance condition, participants were required to magnitude estimate interpoint distances for all possible pairs of points on a simple map. Earlier work has shown that subjective distance judgments $(J)$ are related to the actual distance $(D)$ by a power function with an exponent $(n)$ of close to 1.00 , where $k$ is a scaling constant (Baird, 1970): $J=k D^{n}$. Therefore, power functions were fit to the pair-distance estimates obtained in the present experiment. In the direct mapping condition, participants were required to sketch the map; intersite distances were measured from the drawn maps. Power functions were also fit to these data. The relative accuracy of distances obtained under the two conditions was compared by examining the power function exponents. Veridical or accurate judgments result in an exponent of 1.0, whereas distorted judgments produce either smaller or greater exponents.

Two additional factors were investigated that may in fluence judgment accuracy. First, subjective estimates were obtained either perceptually while the participants viewed the map or from memory after a brief $(10-\mathrm{min})$ or a long (24-h) retention period. Previous research has suggested that judgments made from the memory can differ systematically from those made perceptually (Kerst \& Howard, 1978; Moyer, Bradley, Sorensen, Whiting, \& Mansfield, 1978). For example, Ekman and Bratfisch (1965) found distortion (i.e., an exponent of .78 rather than 1.0) when distance judgments were made from memory. Second, properties of the map itself can lead to judgment error, especially under memory conditions. Howard and Kerst (1981) found a tendency for map sites to form separate clusters in remembered maps. In the present experiment, three different maps were employed that varied in the type and number of clustering cues. The no-cue map depicted a hypothetical college campus with a nearly uniform distribution of building sites. The namecue map was physically identical to the no-cue map, but building sites on the left half of the map were labeled with dormitory names and those on the right were labeled with department names. The name/road-cue maps included a 


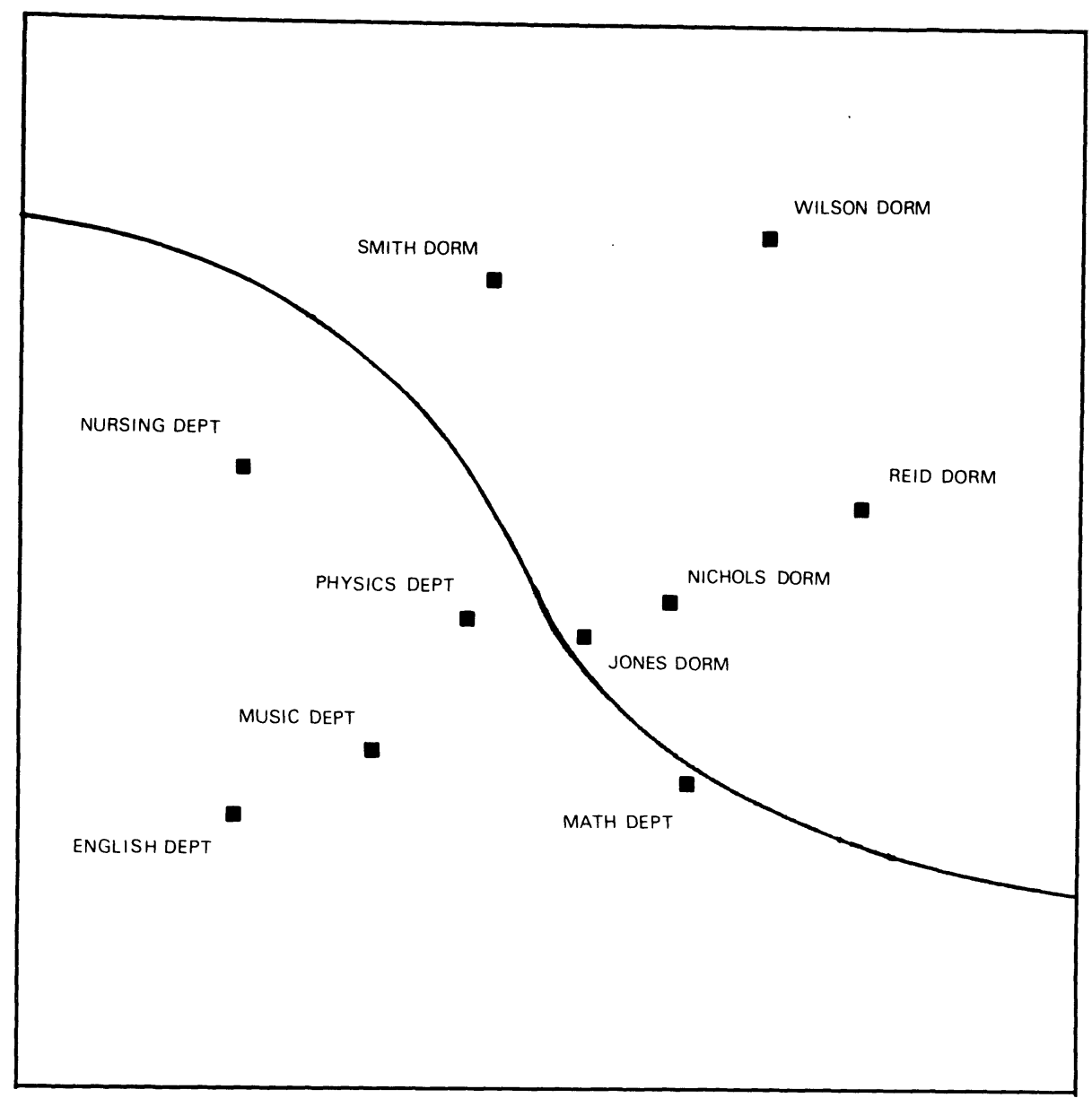

Figure 1. Fictitious college campus map used in the name/road-cue condition. A second map was constructed by substituting Owens for Jones, Johnson for Wilson, Parker for Smith, Coleman for Reid, Turner for Nichols, History for Nursing, Biology for Math, Physical Education for English, Drama for Physics, and German for Music.

campus road that separated the left and right half of the map in addition to the labels used on the name-cue maps.

\section{METHOD}

\section{Participants}

Sixty undergraduate volunteers received course credit in an introductory psychology class for participating.

\section{Design}

Independent variables were (1) judgment method (pair-distance, direct mapping), (2) retention period (perceptual, immediate memory, delayed memory), and (3) map type (no-cue, name-cue, name/road-cue). The dependent variable was the exponent of the power function.

\section{Materials}

Six maps of a fictitious college campus were drawn on $20 \times 20 \mathrm{~cm}$ paper. The map for the name/road-cue condition is shown in Figure 1. The name-cue map was identical to this except that it had no road. The no-cue map was the same as the name-cue map, but the 10 site names were permuted so that the dorms and departmental buildings did not form separate clusters. In order to avoid any artifact due to idiosyncratic properties of the specific names used, a second set of three maps was constructed with different building names. No scale was provided explicitly on the maps.

\section{Procedure}

Six independent groups of 10 individuals were tested. Three groups, one for each of the map types (no-cue, name-cue, and name/road-cue), served in the immediate-memory condition (10-min delay), and three (one for each map type) served in the perceptual condition. The three groups in the perceptual condition returned to the lab after $24 \mathrm{~h}$ to be retested in the delayed-memory condition. Pair-distance judgments were obtained for each of the nine test conditions (three map cue types $\times$ three delays); however, sketched maps were obtained at only the immediate-memory and delayed-memory tests. Individuals were not asked to sketch a map in the perceptual condition. In all, 60 individuals were tested, $\mathbf{3 0}$ for one session and $\mathbf{3 0}$ for two sessions. The two sets of building names occurred equally often within each of the test conditions.

Distance judgments were obtained by presenting pairs of building names on a CRT display in a random order. Responses were entered on the terminal keyboard. For the direct mapping, participants were given a blank piece of paper the same size as the original map $(20 \times 20 \mathrm{~cm})$ and a pencil.

Perceptual condition. The perceptual condition began with a 10-min study period. Participants were instructed to examine the map and to 
pay particular attention to the building locations. Following this, the map was placed on a stand adjacent to the CRT screen where it remained throughout the session. Participants were told that on each trial they would be given the names of two buildings and that they should estimate "the straight-line or direct distance between them relative to the scale of the map, which is 1,000 yards from the right to left edge by 1,000 yards from top to bottom."

After the instructions, participants were given five randomized practice trials with the experimenter present and, after the experimenter left, 180 randomized test trials. The 90 possible ordered pairs of 10 items were each presented twice. The participants initiated each trial and two 5-min breaks were permitted during the experiment.

Delayed-memory condition. Each of the $\mathbf{3 0}$ individuals in the perceptual condition returned after $24 \mathrm{~h}$ to be tested in the delayed-memory condition. The participants understood that they were returning for a related experiment; they were not told that the same maps or procedure would be used. In the delayed-memory session, participants did not see the map at all. Instead, they were given 5 min to "refresh [their] memory of the map [they] had studied the previous day." They were asked to remember as much as they could about the map and to pay particular attention to the locations of the buildings. They were also given a randomized list of the 10 buildings. Participants then received 10 practice trials followed by 180 test trials, as in the perceptual condition. After making their distance judgments, the participants were asked to draw the map. They were told that they could erase and relocate points in drawing their maps.

Immediate-memory condition. The immediate-memory condition was the same as the perceptual session except that the map was removed after the study period and pair-distance estimates were made from memory. The practice and test trials occurred as in the perceptual condition. After completing the distance estimation, the participants were asked to draw the maps as in the delayed-memory condition. Test sessions required approximately $\mathbf{l} \mathrm{h}$ on the average, and participants were debriefed after completing the experiment.

\section{RESULTS}

\section{Psychophysical Analysis}

A geometric mean estimate was determined for each of the 45 building pairs (disregarding order) for each individual for each condition. Psychophysical power functions were then fit to the individual participant data in each condition using standard regression techniques. Reasonable fits were obtained in most cases. The power function failed to account for a significant proportion of the variance at the .05 level in only one of the 90 cases (10 in each of 3 testing $\times 3$ map conditions). Mean correlation coefficients of $.99, .91$, and .88 were obtained for the perceptual, immediate-memory, and delayed-memory conditions, respectively.

A similar analysis was performed on the sketched map data. Coordinates were determined for each site on each of the 60 drawn maps (10 in each of 2 memory $\times 3$ map conditions). Power functions were fit to these data; only one of the 60 functions failed to account for a significant portion of the variance at the .05 level. The mean correlations were .95 for the immediate-memory condition and .92 for the delayed-memory condition.

The power function exponents were the primary dependent variables in the experiment. Three separate analyses of variance (ANOVAs) were performed on these data because of the nonfactorial design employed. First, using data from distance estimates, the perceptual and immediate-memory conditions were compared in a memory condition (perception, immediate-memory) by map cue factorial two-way ANOVA. Second, the perceptual and delayed-memory distance estimate data were compared in a memory condition (perception, delayedmemory) by map cue (no-cue, name-cue, name/road-cue) mixed-design two-way ANOVA with repeated measures on the memory condition factor. Third, possible effects of the response mode were examined in a memory condition (immediate-memory, delayed-memory) by map cue by response mode (distance estimation, sketching) mixed design three-way ANOVA with repeated measures on the response mode factor. The results of these analyses are considered in detail for each independent variable.

\section{Map Clustering Cue}

The main effect of map cue did not approach significance on any of the three ANOVAs. Furthermore, since this factor did not interact significantly with any other factor, the effects of map cue will not be considered further.

\section{Memory Condition}

A mean exponent was determined for each memory and response condition. These data are shown in Figure 2. For the distance estimation data, the perceptual judgments produced a reliably larger exponent than did either of the immediate-memory judgments $[F(1,54)=29.21$, $p<.001]$ or the delayed-memory judgments $[F(1,27)=$ $54.40, p<.001]$. The participants produced more veridical distance estimates in the perceptual condition (mean exponent of 1.09) than in either memory condition (.77 for the immediate, .66 for the delayed).

\section{Response Mode}

Individuals in the two memory conditions responded both by distance estimation and by direct sketching. Possible differences in the accuracy of these data are of

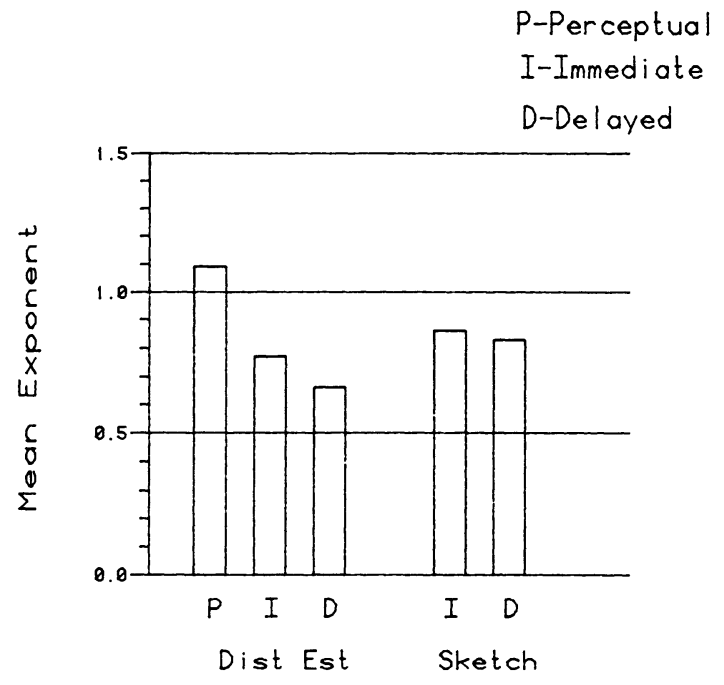

Figure 2. Mean power function exponents for the three memory conditions and two response modes. 
primary interest in the present study. Examination of Figure 2 reveals an overall difference between the mean exponents for these conditions with the sketched maps having a larger and more veridical exponent (mean of .84) than the distance estimates (mean of .72). This difference was statistically significant in the three-way ANOVA described previously $[F(1,54)=23.61, p<.001]$. Furthermore, the effect of response mode was similar at both memory intervals since the memory condition by response mode interaction did not reach statistical significance $[F(1,54)=2.70, p<.10]$. For the immediate-memory condition, 22 of the 30 individuals produced more accurate distances in the sketched map than in their distance estimates ( $p<.01$, binomial test). Similarly, 26 of the 30 participants in the delayed-memory condition had more veridical sketched maps than distance judgments $(p<.001$, binomial test).

\section{DISCUSSION}

Two major findings were revealed in the present study. First, perceptual pair-distance judgments were found to be more nearly veridical than were those made from memory. Second, the results show clearly that when judgments were made from memory, intersite distances on sketched maps were more accurate than the same distances estimated in a pair-distance judgment task. This latter finding is of considerable practical significance since accurate distance estimation from memory is important in many navigation tasks.

The finding that perceptual pair-distance judgments are more accurate than those made from memory is not surprising. However, the observed mean exponent of 1.09 for the perceptual condition is greater than would be expected from previous research. For example, nearly veridical exponents (i.e., 1.00) have been obtained by Baird (1970) and in previous research in our laboratory (Kerst \& Howard, 1978). A closer examination of the present data reveals that the inflated exponent may reflect an effect-albeit, statistically nonsignificant-of the map cluster cue. Perceptual judgments were veridical for the no-cue map (mean exponent of 1.01), but showed increasing distortion for the name cue (1.10) and name/road-cue maps (1.15). Exponents greater than 1.00 indicate a tendency for judges to overestimate the longer distances and/or underestimate the shorter distances, a possible effect of the map clustering cues.

There are three alternative explanations for the second result that maps sketched from memory were more accurate than pair-distance judgments made from memory. First, it is possible that the result simply reflects artifact due to practice. In each condition in which interpoint judgments and sketched maps were obtained, the maps were sketched after the distance estimates were made. To consider this possibility further, an additional group of 10 participants was tested, replicating the immediatememory condition with the no-cue map, but with map sketching occurring before distance estimation. For all 10 individuals, distances on the sketched map were more veridical than those estimated directly, ruling out an explanation based on practice artifact.
A second possibility is that the two tasks, map sketching and distance judgment, call upon two different aspects of spatial knowledge. In this view, map sketching would be based on a more accurate internal representation of the map than would pair-distance judgment. This explanation seems unlikely a priori because of its lack of parsimony. Third, it is possible that both tasks tap the same internal representation, but that corrections or adjustments are made during map sketching which do not occur in pair-distance estimation. For example, when a third point is added to two previously plotted points during map sketching, its location is determined by two interpoint distances rather than by only one. Similarly, when a fourth point is added to three, three constraints exist, and so on. If the participant's memory is not equally accurate for all interpoint distances, then this redundancy provides an opportunity for error correction in map sketching which is not possible in distance estimation. Although the third of these explanations seems more reasonable than the second, neither can be distinguished adequately from the present data. Regardless of which explanation is correct, it is clear that under some conditions (e.g., when spatial knowledge is based on inspection of an unfamiliar map) remembered distances between points are determined more accurately from a sketched map than from intersite distance estimates. This finding has implications for even very simple memory-based navigation tasks. For example, in the present study, a subjective distance involving the full map scale (1,000 yards) would be substantially shorter $(44 \%)$ under distance estimation than on the sketched map.

\section{REFERENCES}

BAIRD, J. C. (1970). A psychophysical analysis of visual space. Oxford: Pergamon Press.

BAIRD, J. C. (1979). Studies of the cognitive representation of spatial relations: I. Overview. Journal of Experimental Psychology: General, 108, 90-91.

Baird, J. C., Merrill, A. A., \& Tannenbaum, J. (1979). Cognitive representation of spatial relations: II. A familiar environment. Journal of Experimental Psychology: General, 108, 92-98.

EkmaN, G., \& BRATFisch, O. (1965). Subjective distance and emotional involvement: A psychological mechanism. Acta Psychologica, 24, 430-437.

GolledGe, R. G., \& Zannaras, G. (1973). Cognitive approaches to the analysis of human spatial behavior. In W. H. Ittelson (Ed.), Environment and cognition. New York: Seminar Press.

HowarD, J. H., JR., \& KerST, S. M. (1981). Memory and perception of cartographic information for familiar and unfamiliar environments. Human Factors, 23, 495-504.

Kerst, S. M., \& Howard, J. H., JR. (1978). Memory psychophysics for visual area and length. Memory \& Cognition, 6, 327-335.

MACKAY, D. B. (1976). The effect of spatial stimuli on the estimation of cognitive maps. Geographical Analysis, 8, 439-452.

Moyer, R. S., Bradley, D. R., Sorensen, M. H., Whiting, J. C., \& MANSFIELD, D. P. (1978). Psychophysical functions for perceived and remembered size. Science, 200, 330-332.

(Manuscript received for publication December 16, 1986.) 